\title{
Diacronie
}

Studi di Storia Contemporanea

$\mathrm{N}^{\circ} 18,2$ | 2014

Le esposizioni: propaganda e costruzione identitaria

\section{Giovanni Tassani, Diplomatico tra le due guerre. Vita di Giacomo Paulucci di Calboli Barone}

\section{Matteo Anastasi}

\section{Q OpenEdition}

Edizione digitale

URL: http://journals.openedition.org/diacronie/1545

DOI: 10.4000/diacronie. 1545

ISSN: 2038-0925

Editore

Association culturelle Diacronie

Notizia bibliografica digitale

Matteo Anastasi, «Giovanni Tassani, Diplomatico tra le due guerre. Vita di Giacomo Paulucci di Calboli Barone », Diacronie [Online], № 18, 2 | 2014, documento 18, Messo online il 01 juin 2014, consultato il 24 septembre 2020. URL : http://journals.openedition.org/diacronie/1545 ; DOI : https://doi.org/ $10.4000 /$ diacronie. 1545 


\section{Diacronie}

N. 18 | 2|2014 Le esposizioni: propaganda e costruzione identitaria

18/

\section{RECENSIONE:}

\section{Giovanni TASSANI, Diplomatico tra le due guerre. Vita di Giacomo Paulucci di Calboli Barone, Firenze, Le Lettere, 2012, pp. 522.} a cura di Matteo ANASTASI *

«Tanto più necessario questo cercare gli uomini quando s'abbia a trattare, come nel caso nostro, di storia politica, soprattutto, di storia dei rapporti politici internazionali: laddove, cioè, non soltanto la personalità generale del singolo politico diplomatico, le sue idee e il suo programma, ma il suo stile d'azione costituisce elemento mai trascurabile nelle vicende ${ }^{1}$. Il monito di un maestro come Federico Chabod riecheggia deciso nella brillante biografia di Giovanni Tassani, Diplomatico tra le due guerre. Vita di Giacomo Paulucci di Calboli Barone, vincitrice della $45^{\mathrm{a}}$ edizione del Premio Acqui Storia. L'opera, collocandosi nel filone degli studi sulla diplomazia italiana del primo Novecento, riesce infatti - con chiarezza espositiva e finezza interpretativa - a ricostruire pulsioni e propositi alla base della condotta diplomatica del funzionario.

Tassani non è nuovo a ricerche di questo genere. L'autore ha infatti iniziato a lavorare sull'archivio dei Paulucci di Calboli già nei primi anni Novanta del secolo scorso, curando la pubblicazione delle memorie di due membri di spicco della familglia forlivese, Raniero² e Fulcieri3 ${ }^{3}$ si è inoltre occupato di storia politica italiana ${ }^{4}$.

L'ultima fatica di Tassani, articolata in dodici punti, ricostruisce vita e azioni di Giacomo Paulucci di Calboli, nato Barone Russo nel 1887, e divenuto marchese nel

${ }^{1}$ CHABOD, Federico, Storia della politica estera italiana dal 1870 al 1896,, Roma-Bari. Laterza, 1971, p. 11.

2 PAUlUCCI DI CALBOLI, Raniero, Parigi 1898. Con Zola per Dreyfus. Diario di un diplomatico, Bologna, Clueb, 1998.

3 PAULUCCI DI CALBOLI, Fulcieri, La patria, l'amore, la guerra. Lettere e scritti 1911-1919, Bologna, Clueb, 1999

4 BAGET BOZZO, Gianni, TASSANI, Giovanni, Aldo Moro. Il politico nella crisi 1962-1973, Firenze, Sansoni, 1983; TASSANI, Giovanni, La terza generazione. Da Dossetti a De Gasperi, tra Stato e rivoluzione, Roma, Lavoro, 1988; ID., L'Italia difficile di Mario Scelba. Sette testimonianze e sette lettere, Soveria Mannelli, Rubbettino, 2006; ID., Il Belpaese dei cattolici. Novecento italiano. Politica e interpretazioni, Siena, Cantagalli, 2010. 
1924, assumendo nome e titoli della famiglia nobiliare della moglie Camilla. Entrato in diplomazia nel 1915, a Berna, parteciperà alla conferenza di pace di Parigi per poi installarsi alla Consulta - l'allora ministero degli Affari Esteri - come segretario di Pietro Tomasi della Torretta prima, e di Carlo Schanzer poi. Con l'avvento del fascismo e il conseguente interim di Mussolini agli Esteri, appena trentacinquenne sarà nominato capo di gabinetto e lavorerà - nella nuova sede di Palazzo Chigi 5 - a stretto contatto con il senatore Salvatore Contarini, diplomatico di carriera dal 1891, segretario generale dal 1920 e fino allora dominus incontrastato della politica estera italiana. Mussolini, del tutto a digiuno di dinamiche internazionali ${ }^{6}$, si affiderà in questa prima fase proprio a Contarini, capace di svolgere - fino alle dimissioni rassegnate nel 1926 una complicata funzione moderatrice sul nuovo capo di governo ${ }^{7}$. Dato il carattere aspro, collerico e poco incline all'indisciplina mussoliniana di Contarini, sarà Paulucci «a trasmettere, mediandole con tatto e pacatezza, le idee del segretario generale al[l'] irruente [...] e apprendista ministro degli Esteri, al fine di costruire una linea di politica estera coerente nel difficile scenario diplomatico e politico del lungo dopoguerra ${ }^{8}$. Come ricorderà in seguito l'allora giovane diplomatico Mario Luciolli:

I primi collaboratori di Mussolini [...] (e innanzi tutto, in ordine di importanza, il segretario generale Contarini e il capo di gabinetto Barone Russo, divenuto poi marchese Paulucci di Calboli) erano diplomatici di carriera, del tutto estranei spiritualmente al fascismo e a qualsiasi altro movimento di piazza e di masse. La loro principale preoccupazione fu di ostentare un conveniente grado di ammirazione per

\footnotetext{
5 Secondo Dino Grandi, Mussolini «si era affrettato a trasferire la sede del Ministero degli Esteri dal Palazzo della Consulta, troppo vicino al Quirinale, a Palazzo Chigi, soprattutto per avere a sua disposizione Piazza Colonna». GRANDI, Dino, Il mio paese. Ricordi autobiografici, Bologna, Il Mulino, 1985, pp. 237-238.

${ }^{6}$ Sull'assenza di un definito programma di politica estera nella prima fase politica di Mussolini, cfr., tra gli altri: DE FELICE, Renzo, Alcune osservazioni sulla politica estera mussoliniana, in ID. (a cura di), L'Italia fra tedeschi e alleati, Bologna, Il Mulino, 1973, p. 58; DI NOLFO, Ennio, Mussolini e la politica estera italiana (1919-1933), Padova, Cedam, 1960, pp. 29-37; KNOX, MacGregor, "Conquest, Foreign and Domestic in Fascist Italy and Nazi Germany», in The Journal of Modern History, 1984, vol. 56, n. 1, pp. 6-20; PASTORELLI, Pietro, «La storiografia italiana del dopoguerra sulla politica estera fascista», in Storia e politica, X, 1971, p. 58; SALVEMINI, Gaetano, Mussolini diplomatico (1922-1932), Bari, Laterza, 1952, pp. 34-35.

7 Cfr. MOSCATI, Ruggero, Gli esordi della politica estera fascista. Il periodo Contarini-Corfù, in TORRE, Augusto et al., La politica estera italiana dal 1914 al 1943, Torino, Eri, 1963, pp. 77117.

8 TASSANI, Giovanni, Diplomatico tra le due guerre. Vita di Giacomo Paulucci di Calboli Barone, Firenze, Le Lettere, 2012, p. 35.
} 
Mussolini e di forzarlo ad avviare la política estera italiana sul binario della diplomazia tradizionale 9 .

Con la fascistizzazione del ministero - iniziata con l'immissione in diplomazia dei cosidetti "ventottisti", i funzionari ammessi per meriti fascisti - Paulucci sarà nominato sottosegretario generale alla Società delle Nazioni, mantendo la carica dal 1927 al 1932, anno dell'epurazione del ministro Dino Grandi e del definitivo attacco mussoliniano all'organizzazione ginevrina. Al rientro, anziché essere resinserito in una carriera oramai lontana dall'apartitizzazione dei primi anni Venti, il duce - ammaliato dall'incontro con Joseph Goebbels, neoministro della Propaganda tedesca - lo eleverà a presidente dell'Ente Nazionale Industrie Cinematrografiche, affidandogli il rilancio dell'Istituto Luce, in crisi finanziaria e organizzativa: «Goebbels mi ha detto che egli ritiene il cinematografo come l'ottavo potere: bisogna potenziare al massimo l'Istituto Luce. È un'arma potentissima che ho affidato alle sue mani»10.

Si trattava di mani tutt'altro che inesperte. Paulucci aveva avuto, infatti, responsabilità manageriali già a Ginevra, garante - in qualità di sottosegretario generale - dell'amministrazione della delegazione italiana presso la Società delle Nazioni. L'esperienza in campo cinematografico si chiuderà nel 1940 con la nomina ad ambasciatore a Bruxelles, cui farà seguito, nel 1943, quella a rappresentante italiano presso la Spagna di Franco, dove alacremente si attiverà per trovare contatti con gli anglo-americani. Dopo il 25 luglio 1943, quando il tramonto calerà sulla lunga giornata dell'Italia fascista, Paulucci cercherà di perorare all'estero la causa del Regno del Sud, quello di Vittorio Emanuele III e di Badoglio. Impegno che non gli eviterà l'epurazione. Differentemente, infatti, da altri diplomatici che svolgeranno un ruolo attivo nella nuova diplomazia italiana, Paulucci si vedrà precluso il rientro a Palazzo Chigi. Su di lui penderanno le pesanti accuse dell'Alto Commissario Aggiunto per l'Epurazione dell'Amministrazione, dalle quali sarà assolto solo nel 1950. Gli ultimi anni, trascorsi da «monarchico in Repubblica» ${ }^{11}$, saranno, infine, caratterizzati dal confronto con don Luigi Sturzo e da un'appassionata attività storiografica vissuta al fianco di due futuri illustri accademici, Mario Toscano e Federico Chabod.

Il volume, fondandosi su una corposa documentazione inedita, costiuisce un'opera preziosa per diverse ragioni. Anzitutto tocca spazi e tempi roventi della storia italiana del XX secolo: dalle complicate trattative versagliesi all'ascesa di Mussolini, dalla guerra civile iniziata nel 1943 alla travagliata ricostruzione. Inoltre, e questo è un

9 LUCIOLLI, Mario, Mussolini e l'Europa. La politica estera fascista, Firenze, Le Lettere, 2009, p. 29.

10 TASSANI, Giovanni, Diplomatico tra le due guerre. Vita di Giacomo Paulucci di Calboli Barone, cit., p. 245.

${ }^{11}$ Ibidem, p. 484 
grande merito, permette di fare luce, non solo su una figura di grande spessore, quale ovviamente quella di Paulucci di Calboli - fino alla biografia di Tassani trattata solo indirettamente dalla storiografia - ma anche sugli uomini che gli operarono al fianco, primo fra tutti Salvatore Contarini, a lungo vero deus ex machina della politica estera italiana, sul quale - causa aridità di fonti specifiche - si è scritto ancora troppo poco ${ }^{12}$. Più in generale, permette di esaminare con rinnovato slancio il ruolo della carriera, vale a dire quella tecnostruttura interna allo Stato - e nell'Italia liberale volutamente scevra da ideologie o correnti politiche - che con Mussolini si trasformerà progressivamente in uno strumento al servizio del fascismo e del suo capo, divenendo «diplomazia di partito ${ }^{13}$.

Nondimeno, l'opera fornisce un'utilissima analisi sulla genesi della propaganda fascista che vide nell'Istituto Luce, ottimamente ristrutturato da Paulucci, il volano in grado di conferire, internamente e all'estero, prestigio al regime tramite produzioni di grande successo, quali Scipione l'Africano di Carmine Gallone ${ }^{14}$ e Condottieri di Luigi Trenker ${ }^{15}$.

12 La documentazione edita e specificamente dedicata a Contarini si riduce pressoché esclusivamente alla datata biografia di Roberto Cantalupo, scritta con lo pseudonimo di Legatus (Vita diplomatica di Salvatore Contarini. Italia fra Inghilterra e Russia, Roma, Sestante, 1947), peraltro da assumere con cautela a causa della scarsa attendibilità di alcune informazioni riportatevi. Esigue notizie sul senatore sono altresì rintracciabili - oltreché in diari e fonti memorialistiche - in libri "di colore" quali, tra gli altri: QUARONI, Pietro, Valigia diplomatica, Milano, Garzanti, 1956; VITA-FINZI, Paolo, Giorni lontani. Appunti e ricordi, Bologna, Il Mulino, 1989.

13 GRASSI ORSINI, Fabio, La diplomazia fascista, in AA.VV., Il regime fascista. Storia e storiografia, Roma-Bari, Laterza, 1995, p. 287.

${ }^{14}$ GALLONE, Carmine, Scipione l'Africano, Consorzio Scipione-ENIC, Italia, 1937, 83'.

15 TRENKER, Luis, Condottieri, ENIC-Tobis Filmkunst, Italia-Germania, 1937, 88'. 


\section{* L'autore}

Matteo Anastasi si è laureato (Bachelor's Degree) c on lode in Scienze Storiche presso l'Università Europea di Roma. Attualmente sta completando gli studi magistrali (Master's Degree) in Relazioni Internazionali presso la Luiss Guido Carli, sotto la guida del prof. Francesco Perfetti. Si occupa di storia politica e diplomatica italiana.

URL: < http://www.studistorici.com/progett/autori/\#Anastasi >

\section{Per citare questo articolo:}

ANASTASI, Matteo, «Recensione: Giovanni TASSANI, Diplomatico tra le due guerre. Vita di Giacomo Paulucci di Calboli Barone, Firenze, Le Lettere, 2012, pp. 522.», Diacronie. Studi di Storia Contemporanea : Le esposizioni: propaganda e costruzione identitaria, 29/6/2014,

URL: < http://www.studistorici.com/2014/6/29/anastasi_numero_18/ >

Diacronie Studi di Storia Contemporanea $\sqrt{3}$ www.diacronie.it

Risorsa digitale indipendente a carattere storiografico. Uscita trimestrale. redazione.diacronie@hotmail.it

Comitato di redazione: Jacopo Bassi - Luca Bufarale - Elisa Grandi - Deborah Paci - Fausto Pietrancosta - Matteo Tomasoni - Luca Zuccolo

Diritti: gli articoli di Diacronie. Studi di Storia Contemporanea sono pubblicati sotto licenza Creative Commons 2.5 . Possono essere riprodotti a patto di non modificarne i contenuti e di non usarli per fini commerciali. La citazione di estratti è comunque sempre autorizzata, nei limiti previsti dalla legge. 